\title{
Motion and kinematics parameters of frozen-thawed bovine spermatozoa assessed by Biovis CASA
}

\author{
PK Pathak, AJ Dhami, DV Chaudhari and JA Patel
}

Received: 15 February 2019 / Accepted: 12 March 2019 / Published online: 22 June 2019

(c) Indian Dairy Association (India) 2019

\begin{abstract}
This study was carried out on semen of nine healthy mature breeding bulls ( 3 each of Gir, Surti and Murrah breed) to evaluate the motion characteristics and kinematics of their frozenthawed sperms by using computer assisted semen analyzer (CASA). A total of 24 ejaculates from each breed were frozen in Tris fructose yolk glycerol extender using biofreezer. The motion characteristics of frozen-thawed semen assessed by CASA revealed same pattern as of fresh semen. The mean values of total motile vs. immotile sperms in Gir, Surti and Murrah bulls' frozen-thawed semen were $58.22 \pm 1.71$ vs. $41.95 \pm 1.71,64.44 \pm 1.58$ vs. $35.56 \pm 1.58$, and $67.84 \pm 1.54$ vs. $31.73 \pm 1.62 \%$, respectively. The corresponding mean values of rapid, slow and non-progressive motile sperm in Gir, Surti and Murrah bulls' semen were 17.93 \pm 1.56 , $17.13 \pm 2.49$ and $21.47 \pm 2.70 \% ; 23.90 \pm 1.70,30.64 \pm 2.06$ and $30.47 \pm 2.25 \%$; and $16.23 \pm 1.24,16.70 \pm 1.58$ and $15.90 \pm 1.52 \%$, respectively. The overall mean velocity/ kimematics parameters based on total motile sperms of Gir, Surti and Murrah bulls were: average path velocity (VAP) $42.14 \pm 1.21,40.15 \pm 1.58$ and $42.41 \pm 1.62$
\end{abstract}

PK Pathak, AJ Dhami $(\bowtie)$ DV Chaudhari and JA Patel Department of Veterinary Gynaecology \& Obstetrics College of Veterinary Science \& Animal Husbandry Anand Agricultural University, Anand-388 001, Gujarat, India Email: ajdhami@aau.in, Mobile:+919898262498 $\mu \mathrm{m} / \mathrm{s}$; curvilinear velocity (VCL) $78.22 \pm 2.01,76.52 \pm 2.31$ and $80.25 \pm 2.25 \mu \mathrm{m} / \mathrm{s}$, straight line velocity (VSL) $35.60 \pm 1.18,33.69 \pm 1.59$ and $36.19 \pm 1.58 \mu \mathrm{m} / \mathrm{s}$; linearity $45.21 \pm 1.62,44.38 \pm 2.05$ and $44.96 \pm 1.35 \%$; straightness $78.53 \pm 1.17,78.75 \pm 1.40$ and $80.24 \pm 1.13$ $\%$; wobbling index $57.86 \pm 1.69,56.78 \pm 1.88$ and $56.55 \pm 1.29 \%$; beatcross frequency (BCF) $11.77 \pm 0.67,12.08 \pm 0.72$ and $12.64 \pm 0.86 \mathrm{hz}$; amplitude of lateral head displacement (ALH) $2.18 \pm 0.17,2.30 \pm 0.15$ and 2.35 $\pm 0.15 \mu \mathrm{m}$; dancing frequency (DNC) $185.08 \pm 15.96$, $183.90 \pm 14.22$ and $198.44 \pm 15.64 \mu \mathrm{m}^{2} / \mathrm{s}$, and dancing mean (DNM) $4.96 \pm 0.51,5.35 \pm 0.54$ and $5.32 \pm 0.51 \mu \mathrm{m}^{2} / \mathrm{s}$, respectively. None of these traits differed significantly between breeds/ species or between bulls in any of the breed. The sperm velocity parameters recorded based on progressively motile sperms revealed almost similar pattern and variation to those found based on total motile sperms, but the values of all velocity parameters for progressive motile sperms were higher than those of total motile sperms in all three breeds when assessed through CASA. It was concluded that frozen-thawed sperm of cattle and buffalo breed show identical CASA traits.

Keywords: Cow bull, Buffalo bull, CASA, Frozen-thawed semen, Sperm motion characteristics

\section{Introduction}

As compared to computer assisted semen analyzer (CASA), the conventional subjective evaluation of bovine semen is relatively inaccurate, imprecise, time consuming (Ventman et al. 1988; Christensen et al. 2005; Amann and Waberski, 2014), and depends on the skill of the investigator (Knuth et al. 1989; Patel and Dhami, 2016). Characterization of semen based on motility, velocity and morphology could improve methods for assessing bull fertility (Kathiravan et al. 2005; Ramachandran et al. 2007). CASA, therefore, has been introduced mainly to save time, to avoid subjective errors in evaluation, to improve the accuracy of data collection, and to gather population values on sperm kinematics (Johnson et al. 1996; Verstegen et al. 2002). The kinematic values determined for each spermatozoon cover the velocity of movement, viz., curvilinear velocity (VCL), straight line velocity (VSL) and average path velocity (VAP), the width of the sperm head's trajectory and frequency of the change in direction of the sperm head (Mortimer et al. 1990) and thus provide quantitative 
assessment of sperms of different species and individual within the breed/species. Further, the seminal attributes of cattle and buffalo bulls are distinct, yet the extender, freezing-thawing protocol and evaluation technique have remained the same for decades for both the species. Therefore, the objective of present study was to quantitatively compare the motility and kinematics of frozen-thawed sperms of Gir, Surti and Murrah bulls using CASA to know the possible utility of CASA in differential evaluation of sperm quality of three breeds.

\section{Materials and methods}

This study was carried out on semen of nine healthy mature breeding bulls (3 each of Gir, Surti and Murrah breed), aged 5-8 years, at the College of Veterinary Science, AAU, Anand-388 001 during winter season of year 2017-18. All the bulls were in good health and under optimal veterinary care. They were maintained in nearly identical nutritional and managerial conditions throughout the period of study with twice a week semen collection schedule. Semen was collected using artificial vagina from each bull in the morning hours between 7.30 and $8.30 \mathrm{hr}$ over a dummy buffalo bull. Ejaculates collected at weekly interval were used for this study. A total of 24 ejaculates from each breed (8/bull) were studied. Immediately after collection, the ejaculates were evaluated for various seminal attributes, and those (24/breed) with more than $75 \%$ initial motility were soon extended at $34^{\circ} \mathrm{C}$ with Tris-citric acid-fructose-egg yolk-glycerol (TFYG) extender keeping 80 million sperm per $\mathrm{ml}$.

The extended semen was soon filled and sealed in French mini straws by IS4 machine (IMV, France), cooled to $5^{\circ} \mathrm{C}$, equilibrated for $4 \mathrm{hrs}$ and frozen in liquid nitrogen vapour using a bio-freezer (IMV, France) employing standard freezing protocol for bovine. The straws were thawed next day in water bath at $37^{\circ} \mathrm{C}$ for 30 second and post-thaw motility was assessed subjectively by the investigator using routine microscopic evaluation. The thawed semen was also soon assessed for sperm motion characteristics by Biovis CASA (Expert Vision Lab Pvt. Ltd. Mumbai). For this $50 \mu 1$ of thawed semen was placed on grease-free clean sterile ordinary glass-slide, covered with a cover slip, and was examined under $40 \mathrm{X}$ of a phase contrast microscope with biotherm connected to a CCTV screen. The analysis set up was selected as per the manufacturer's instruction. The CASA traits studied as per software included total motile sperms (grouped as rapid, slow and non-progressive motile sperms), immotile sperms, average path velocity (VAP), curvilinear velocity (VCL), straight line velocity (VSL), straightness (STR), linearity (LIN), wobbling (WOB), beat-cross frequency (BCF), amplitude of lateral head displacement (ALH), dancing frequency (DNC) and dancing mean (DNM). The data generated on sperm quality traits of frozenthawed semen were analyzed statistically for ANOVA using standard statistical package on SPSS software version 20.00 for each breed and the breed differences were compared by DMRT (Snedecor and Cochran, 1994).

\section{Results and discussion}

\section{Sperm motility profile}

The mean post-thaw sperm motility ratings recorded by subjective assessment for Gir, Surti and Murrah bulls semen by microscopic evaluation was $53.29 \pm 1.56,58.33 \pm 1.43$ and $59.58 \pm 1.20$ per cent, respectively. It was significantly $(\mathrm{p}<0.01)$ lower in Gir bulls than Surti and Murrah bulls. Moreover, the bull variation was significant only among buffalo breeds. Similar significant breed and species variation in semen freezability has been reported by earlier workers with better results in buffalo (Dhami et al. 1998; Shelke and Dhami, 2001; Chaudhary et al. 2017), although it is in contrast to reports available for cattle and buffalo semen freezability (Rhodes et al. 1985; Dhami et al. 1990).

The CASA analysis of post-thawed semen revealed the overall mean values of total motile sperms for Gir, Surti and Murrah bulls as $58.22 \pm 1.71,64.44 \pm 1.58$ and $67.84 \pm 1.54 \%$, respectively. It was significantly $(\mathrm{p}<0.01)$ higher in buffalo bulls than Gir, and was also higher than the subjective rating through microscopic evaluation by the investigators. Amongst the CASA traits, the immotile and non-progressive motile sperm per cent were lower in semen of Murrah bulls than Surti and Gir bulls, while total motile, rapid progressive motile and slow progressive motile sperm per cent were higher in Gir bulls than Surti and Murrah bulls (Table 1). Similar higher values of post-thawed sperm motility traits in buffalo bull than cow bull semen were reported earlier by Patel et al. (2010) using Hamilton Thorne CASA.

Some of the earlier studies also reported similar type of sperm motility ratings of frozen-thawed semen of farm animals using different versions of CASA machines (Rasul et al. 2001; Mandal et al. 2003; Ramachandran et al. 2007; Kathiravan et al. 2005, 2008; Patel and Dhami, 2013; Patel and Dhami, 2016; RodriguezMontaña and Roa-Guerrero, 2017; Kumar et al. 2018). However, in many of these reports only a few of the CASA traits were reported at a time, rather than exhaustive evaluation as done in the present study. Our results on CASA based motility rating of frozen-thawed bull and buffalo bull sperms are in accordance with some of these reports, particularly of Karthikeya (2003), Kathiravan et al. (2005), Ramachandran et al. (2007), and Patel and Dhami $(2013,2016)$. Breed variation in the CASA quality of sperm has also been reported by Haflock et al. (2006). However, the percentages of total motile and progressive motile sperms varied among different studies due to variation in the breeds of bulls, initial and post-thaw quality of semen, extender used, postthaw longevity of sperms, software and make of CASA machines used. Our rating for post-thaw semen with Biovis CASA was far higher than that reported by Kumar et al. (2018) using same version of CASA, but was lower than that found using Hamilton Thorne CASA by Kathiravan et al. (2005), and Patel and Dhami (2013, 2016). 


\section{Sperm velocity / kinematics}

The findings of CASA analysis of post-thawed semen for sperm velocity and kinematics parameters based on total motile sperms and progressively motile sperms are presented in Tables 2 and 3 , respectively.

In frozen-thawed bulls' semen, the overall mean values of various

Table 1 Average $( \pm \mathrm{SE})$ sperm motility parameters of frozen-thawed semen of Gir cattle and Surti and Murrah buffalo bulls assessed by CASA

\begin{tabular}{lllc}
\hline Seminal attributes & Breeds of bulls & & \\
\cline { 2 - 4 } & Gir(n=24) & Surti (n=24) & Murrah (n=24) \\
\hline Total motile sperm (\%) & $58.22^{x} \pm 1.71$ & $64.44^{\mathrm{y}} \pm 1.58$ & $67.84^{y^{*} \pm 1.54}$ \\
Rapid progressive motile sperm (\%) & $17.93 \pm 1.56$ & $17.13 \pm 2.49$ & $21.47 \pm 2.70$ \\
Slow progressive motile sperm (\%) & $23.90^{\mathrm{x}} \pm 1.70$ & $30.64^{\mathrm{y}} \pm 2.06$ & $30.47^{\mathrm{y}} \pm 2.25$ \\
Non-progressive motile Sperm (\%) & $16.23 \pm 1.24$ & $16.70 \pm 1.58$ & $15.90 \pm 1.52$ \\
Immotile sperm(\%) & $41.95^{\mathrm{y}} \pm 1.71$ & $35.56^{\mathrm{x}} \pm 1.58$ & $31.73^{\mathrm{x}} \pm 1.62$ \\
\hline
\end{tabular}

$\mathrm{N}=$ Number of bull. Means bearing uncommon superscripts within the row differ significantly $(\mathrm{p}<0.05)$. Bull variation was not significant for any of the traits in any of the breeds.

Table 2 Average ( \pm SE) sperm kinematics parameters of total motile sperms of frozen-thawed semen of Gir cattle and Surti and Murrah buffalo bulls assessed by CASA

\begin{tabular}{lllc}
\hline Seminal attributes & Breeds of bulls & & Murrah $(\mathrm{n}=24)$ \\
\cline { 2 - 4 } & Gir(n=24) & Surti $(\mathrm{n}=24)$ & $42.41 \pm 1.62$ \\
\hline Average path velocity (VAP, $\mu \mathrm{m} / \mathrm{s})$ & $42.14 \pm 1.21$ & $40.15 \pm 1.58$ & $80.25 \pm 2.25$ \\
Curvilinear velocity (VCL, $\mu \mathrm{m} / \mathrm{s}$ ) & $78.22 \pm 2.01$ & $76.52 \pm 2.31$ & $36.19 \pm 1.58$ \\
Straight line velocity (VSL, $\mu \mathrm{m} / \mathrm{s}$ ) & $35.60 \pm 1.18$ & $33.69 \pm 1.59$ & $44.96 \pm 1.35$ \\
Linearity (LIN, \%) & $45.21 \pm 1.62$ & $44.38 \pm 2.05$ & $80.24 \pm 1.13$ \\
Straightness (STR, \%) & $78.53 \pm 1.17$ & $78.75 \pm 1.40$ & $56.55 \pm 1.29$ \\
Wobbling index (WOB, \%) & $57.86 \pm 1.69$ & $56.78 \pm 1.88$ & $12.64 \pm 0.86$ \\
Beat-cross frequency (BCF, hz) & $11.77 \pm 0.67$ & $12.08 \pm 0.72$ & $2.35 \pm 0.15$ \\
Amplitude of lateral head displacement (ALH, $\mu \mathrm{m})$ & $2.18 \pm 0.17$ & $2.30 \pm 0.15$ & $198.44 \pm 15.64$ \\
Dancing frequency $\left(\mathrm{DNC}, \mu \mathrm{m}^{2} / \mathrm{s}\right)$ & $185.08 \pm 15.96$ & $183.90 \pm 14.22$ & $5.32 \pm 0.51$ \\
Dancing mean $\left(\mathrm{DNM}, \mu \mathrm{m}^{2} / \mathrm{s}\right)$ & $4.96 \pm 0.51$ & $5.35 \pm 0.54$ & \\
\hline
\end{tabular}

$\mathrm{N}=$ Number of bull. No significant variation between breeds or between bulls within the breed was found for any of the traits $(\mathrm{p}>0.05)$.

Table 3 Average ( \pm SE) sperm kinematics parameters of only progressively motile sperms of frozen-thawed semen of Gir cattle and Surti and Murrah buffalo bulls assessed by CASA

\begin{tabular}{|c|c|c|c|}
\hline \multirow[t]{2}{*}{ Seminal attributes } & \multicolumn{3}{|c|}{ Breeds of bulls } \\
\hline & $\operatorname{Gir}(n=24)$ & Surti $(n=24)$ & Murrah $(n=24)$ \\
\hline Average path velocity (VAP, $\mu \mathrm{m} / \mathrm{s}$ ) & $48.88 \pm 1.32$ & $44.85 \pm 1.57$ & $47.22 \pm 1.74$ \\
\hline Curvilinear velocity (VCL, $\mu \mathrm{m} / \mathrm{s}$ ) & $83.11 \pm 2.40$ & $78.85 \pm 2.56$ & $82.62 \pm 2.53$ \\
\hline Straight line velocity (VSL, $\mu \mathrm{m} / \mathrm{s}$ ) & $44.82 \pm 1.22$ & $40.77 \pm 1.53$ & $43.15 \pm 1.58$ \\
\hline Linearity (LIN, \%) & $54.66 \pm 1.34$ & $52.93 \pm 1.81$ & $53.09 \pm 1.06$ \\
\hline Straightness (STR, \%) & $90.83 \pm 0.60$ & $90.21 \pm 0.69$ & $90.73 \pm 0.38$ \\
\hline Wobbling index (WOB, \%) & $59.75 \pm 1.39$ & $58.10 \pm 1.72$ & $57.93 \pm 0.98$ \\
\hline Beat-cross frequency (BCF, hz) & $14.30 \pm 0.74$ & $14.13 \pm 0.75$ & $15.06 \pm 0.83$ \\
\hline $\begin{array}{l}\text { Amplitude of lateral head } \\
\text { displacement }(\mathrm{ALH}, \mu \mathrm{m})\end{array}$ & $2.25 \pm 0.19$ & $2.30 \pm 0.16$ & $2.38 \pm 0.17$ \\
\hline Dancing frequency $\left(\mathrm{DNC}, \mu \mathrm{m}^{2} / \mathrm{s}\right)$ & $191 \pm 16.41$ & $184 \pm 14.12$ & $203 \pm 16.33$ \\
\hline Dancing mean (DNM, $\left.\mu \mathrm{m}^{2} / \mathrm{s}\right)$ & $4.43 \pm 0.37$ & $4.60 \pm 0.35$ & $4.70 \pm 0.36$ \\
\hline
\end{tabular}

$\mathrm{N}=$ Number of bull. No significant variation between breeds or between bulls within the breed was found for any of the traits $(\mathrm{p}<0.05)$. 
sperm parameters recorded for total motile sperms, like average path velocity (VAP), curvilinear velocity (VCL) and straight line velocity (VSL), linearity (LIN), straightness (STR), wobbling index (WOB), and even the mean values of BCF, ALH, DNC and DNM (Table 2) revealed that none of these traits differed significantly between breeds/species or between bulls in any of the breed. However, the mean values of VAP, VCL, and VSL were little higher in Murrah and Gir bulls than Surti bulls, those of LIN and WOB were higher in Gir compared to Surti and Murrah buffalo, while STR was higher in Murrah than Surti and Gir bulls. Similar observations of post-thawed sperms of different breeds of cattle were also reported earlier by Patel et al. (2010).

The velocity / kinematic parameters of only progressive motile sperms of frozen-thawed semen when compared with those of total motile sperms (Table 3) revealed that the trend and differences were similar to those observed based on total motile sperms (Table 2), but the values of velocity and kinematics attributes for progressive motile sperms were higher than those for total motile sperms with significant $(\mathrm{p}<0.05)$ difference in VAP, VSL, linearity and straightness. Again, none of these traits differed significantly between breeds/species or between bulls in any of the breed. Comparable attributes and their values for frozenthawed semen were also reported by earlier workers (Rasul et al. 2001; Kathiravan et al. 2005; Koonjaenak et al. 2006; Ramachandran et al. 2007; Patel and Dhami, 2016). However, the absolute values of these velocity and kinematics traits varied among different studies probably due to aforesaid reasons. The information generated on different sperm quality traits using Biovis CASA were far higher than those reported by Kumar et al. (2018) using same version of CASA, and fell in the range of results of most of the other studies using advance costly versions of Hamilton Thorne CASA (Karthikeya, 2003; Kathiravan et al. 2005; Ramachandran et al. 2007; Patel and Dhami, 2016).

Rasul et al. (2001) reported linear motility of frozen-thawed buffalo sperm as $79.6 \pm 1.4 \%$, VCL $69.4 \pm 2.0 \mu \mathrm{m} / \mathrm{s}$ and ALH $1.7 \pm 0.2 \mu \mathrm{m}$. Koonjaenak et al. (2006) reported mean values of linear motile $(\%), \operatorname{VAP}(\mu \mathrm{m} / \mathrm{s}), \operatorname{VSL}(\mu \mathrm{m} / \mathrm{s}), \operatorname{VCL}(\mu \mathrm{m} / \mathrm{s})$ and $\operatorname{ALH}(\mu \mathrm{m})$ as $48.8 \pm$ $1.9,90.7 \pm 1.0,87.9 \pm 1.0,128.0 \pm 1.2$ and $3.5 \pm 0.1$, respectively, in frozen-thawed semen of buffalo bulls. Ramachandran et al. (2007) reported the means for computer assisted frozen-semen analysis parameters of Sahiwal - the zebu - bulls like per cent motility, $\operatorname{VAP}(\mu \mathrm{m} / \mathrm{sec}) \operatorname{VSL}(\mu \mathrm{m} / \mathrm{sec}), \operatorname{VCL}(\mu \mathrm{m} / \mathrm{sec}), \operatorname{LIN}(\%)$, and ALH $(\mu \mathrm{m})$ as $60.17 \pm 1.24,64.84 \pm 1.50,42.03 \pm 1.05,92.74 \pm 2.51,59.39 \pm 1.50$ and 6.99 \pm 0.24 , respectively. Patel et al. (2013) and Patel and Dhami (2016) evaluated frozen-thawed semen from Jafarabadi, Mehsana and Crossbred (HF x K) bulls through Hamilton Thorne CASA and observed that the mean values of motile and progressively motile spermatozoa, VAP, VSL, and VCL did not differ significantly between three breeds. However, the other velocity parameters, viz., ALH $(\mu \mathrm{m}), \mathrm{BCF}(\mathrm{Hz}), \mathrm{STR}(\%)$, linearity (\%), elongation (\%) and sperm area $\left(\mu \mathrm{m}^{2}\right)$ recorded for the sperm of Jafarabadi bulls $(6.72 \pm 0.19,34.05 \pm 0.46,74.70 \pm 1.02,44.37 \pm 1.01,50.73 \pm 0.93$ and
$10.83 \pm 0.17$, respectively) were similar to Mehsana bulls, but differed from crossbred bulls $(7.86 \pm 0.14,31.88 \pm 0.39,69.57 \pm 0.67$, $38.10 \pm 0.72,43.83 \pm 0.97$, and $12.03 \pm 0.16$, respectively). The rapidly motile, medium, slow motile and static sperm in the frozen-thawed semen were similar in all three breeds. The present results with cheaper Biovis CASA employing ordinary slides also concurred with the above reports using advanced version of Hamilton CASA.

\section{Conclusions}

The present findings using Biovis CASA indicated that the postthawed sperm motion characteristics, viz., motile, progressive motile sperm, VAP, VSL, VCL, ALH, BCF, straightness, linearity, elongation, wobbling and dancing were similar in bulls of Gir, Surti and Murrah breeds, hence Biovis CASA may be used to assess sperm kinematics of frozen-thawed bovine semen as an alternative to costly CASA versions.

\section{Acknowledgements}

We thank the Dean of the College and authorities of Anand Agricultural University, Anand for the facilities provided for this work.

\section{References}

Amann RP, Waberski D (2014) Computer-assisted sperm analysis (CASA): capabilities and potential developments. Theriogenology 81: 5-17

Chaudhary PJ, Dhami AJ, Chaudhari DV, Hadiya KK, Patel JA (2017) Comparative study of Gir cattle and Surti buffalo bulls semen under middle Gujarat climate. Indian J Vet Sci Biotech 13(1): 56-61

Christensen P, Boelling D, Pedersen KM, Korsgaard IR, Jensen J (2005) Relationship between sperm viability as determined by flow cytometry and nonreturn rate of dairy bulls. J Androl 26: 98-106

Dhami AJ, Panchal MT and Kodagali SB (1990) Comparative biochemical and enzymatic study of Jersey, crossbred and Surti buffalo semen with reference to freezability and fertility. Indian J Anim Res 24(1): $1-8$

Hoûack G, Opsomer G, Rijsselaere T, Van Soom A, Maes D, Kruif de A, Duchateau L (2007) Comparison of computer assisted sperm motility analysis parameters in semen from Belgian Blue and Holstein-Friesian bulls. Reprod Dom Anim 42: 153-161

Johnson J, Boone W, Blackhurst D (1996) Manual versus computerautomated semen analysis. Part I. Comparison of counting chambers. Fertil Steril 65: 150-155

Karthikeya G (2003) Computer automated motility and morphometric analysis of bull and buck spermatozoa. MVSc Thesis, Tamil Nadu University of Veterinary \& Animal Sciences, Chennai, India

Kathiravan P, Kalatharan J, Edwin MJ, Veerapandian C (2008) Computer automated motion analysis of crossbred bull spermatozoa and its relationship with in vitro fertility in zona-free hamster oocytes. Anim Reprod Sci 104: 9-17

Kathiravan P, Kalatharan J, John Edwin M, Veerapandian C (2005) Postthaw sperm motion characteristics of different crossbred bull spermatozoa assessed by computer assisted semen analyzer. J Remount Vet Corps 44: 33-38

Knuth UA, Neuwinger J, Nieschlag E (1989) Bias of routine semen analysis by uncontrolled changes is laboratory environment - detection by 
long term sampling of monthly means for quality control. Intl $\mathrm{J}$ Androl 12: 373-383

Koonjaenak S, Kunavongkrit A, Chanatinart V, Sirivaidyapong S, Pinyopumimintr T, Rodriguez-Martinez H (2006) Semen quality of Thai swamp buffalo artificial insemination bulls: comparison of production data from 1988-1993, 2001-2004 and 2004-2005. Buffalo J 22: 1-9

Kumar S, Kumar A, Singh AK, Honparkhe M, Singh P, Malhotra P (2018) Improvement in post-thaw semen quality by minimizing the lipid peroxidation following herbal treatment in sub fertile buffalo bulls. The Pharma Innovation 7(5, Part D): 240

Mandal DK, Nagpaul PK, Gupta AK (2003). Motion characteristics of Murrah buffalo bull spermatozoa in various seasons and its relationship with functional integrity of the plasmallema. Theriogenology 60(2): 349-358

Mortimer D, Keel BA, Webster BW (1990) Objective analysis of sperm motility and kinematics. Eds. Handbook of the Laboratory Diagnosis and Treatment of Infertility. Boca Raton, Fla. CRC Press, pp. 97133.

Patel JB, Dhami AJ (2016) Computer assisted sperm analysis of fresh and frozen-thawed buffalo semen and their interrelationship. Indian $\mathrm{J}$ Anim Res 50(1): 8-13

Patel JB, Dhami AJ (2013) Computer assisted sperm analysis of fresh and frozen-thawed HF x Kankrej (F1) bulls' semen and their interrelationship. Indian J Anim Res 47(4): 315-320

Patel NP, Patel SH, Devanand CP (2010) Comparative studies on the assessment of the bovine sperm motility by conventional method and CASA System (HTM IVOS). Proc. XXVI Annual Convention of
ISSAR and International Symposium, GBPUA \& T, Pantnagar,UP, India 10-12 Nov., p. 11

Ramachandran N, Verma GS, Gupta AK, Raina VS, Mohanty TK, Singh MK (2007) Prediction of fertility based on frozen-thawed seminal traits in Sahiwal bulls. Indian J Anim Sci 77(8): 884-887

Rasul Z, Ahmad N, Anzar M (2001) Changes in motion characteristics, plasma membrane integrity, and acrosome morphology during cryopreservation of buffalo spermatozoa. J Androl 22: 278-283

Rhodes F, Galina CS, Duchateau A, Sato C (1985) An investigation into the properties of bovine semen in the Mexican tropics. World Rev Anim Prod 21(2): 15-19

Rodriguez-Montaña D, Roa-Guerrero E (2017) Objective assessment of bull sperm motility parameters using computer vision algorithms. African J Biotech 16(37): 1871-1881

Shelke VB, Dhami AJ (2001) Comparative evaluation of physicomorphological attributes and freezability of semen of Gir cattle (Bos indicus) and Jafarabadi buffalo (Bubalus bubalis) bulls. Indian J Anim Sci 71: 319-324

Snedecor GW, Cochran WG (1994) Statistical Methods. $14^{\text {th }}$ edn. Oxford and IBH Publishing House, New Delhi, India

Vantman D, Koukoulis G, Dennison L, Zinaman M, Sherins RJ (1988) Computer-assisted semen analysis: evaluation of method and assessment of the influence of sperm concentration on linear velocity determination. Fertil Steril 49: 510-515

Verstegen J, Iguer-Ouada M, Onclin K (2002) Computer assisted semen analyzers in andrology research and veterinary practice. Theriogenology 57(1): 149-179 